\title{
SYNTHESIS OF CAM MECHANISMS WITH INPUT TRANSLATION, FLAT AND PROFILED FOLLOWER
}

\author{
Blagoy Ka Ilieva Paleva-Kadiyska* \\ Department Mechanical engineering and technologies, \\ Faculty of engineering, Neofit Rilski South-West University, \\ Blagoevgrad 2700, Bulgaria
}

[Received: 28 March 2019. Accepted: 30 April 2020]

\begin{abstract}
A mathematical model is derived for synthesis of CAM mechanisms with input translation, flat and profiled follower. Considering the pressure angle the basic parameters of the mechanism are determined. Several equations are derived: of the curve of centers of the processing tools and the pitch curves of the roll, the CAM profiles, and their common evolute. An example is given illustrating the application of the mathematical model under three different possible synthesis requirements.
\end{abstract}

KEY WORDS: CAM mechanism, synthesis, pressure angle.

\section{INTRODUCTION}

The widespread use of CAM mechanisms is due to their compactness, the ability to realize almost all the laws of motion and the ease of incorporation into mechanical systems of machines [1,2].

This has led to their use in various machines in many industries: textile, food processing, printing, aircraft, transport, agricultural, etc. [3].

There are an endless number of CAM mechanisms, with which is possible to generate a law of motion, unlike linkages [4]. Four-bar mechanisms can be considered as an instance of CAM mechanisms. Therefore, their ability to generate functions is significantly lower than the CAM mechanisms [5].

Dozens of publications are mostly related to the synthesis of disk CAM mechanisms [6]. Relatively smaller is the number of posts dedicated to translating CAM mechanism, also known as linear CAM or flat-faced CAM [7-9].

The design of CAMs with input translation is less well studied, despite their widespread use in various copiers, electric switches [10] and other applications [11]. Even less have been studied the problems associated with the design of mechanisms containing profiled output link, also known as inverse CAM mechanisms or profiledfollower mechanisms [12-14].

\footnotetext{
*Corresponding author e-mail: paleva-kadiyska.bl@abv.bg
} 
The aim of the present study is synthesis of CAM mechanisms with input translation, both flat and profiled follower (slider). Synthesis includes the poorly studied problem of defining the basic geometric parameters of these mechanisms and the CAMs profiling.

\section{Mechanisms With InPUT and OUTPUt Translation}

Probably the first CAM used by man is the wedge - for woodcutting, stone crushing, lifting loads. At an angle $\theta<45^{\circ}$ of wedge sharpness, input force $F_{\sigma}$ is multiplied in output force $F_{S}=F_{\sigma} / \tan \theta$, if friction is not taken into account (Fig. 1a). Therefore, this angle, called pressure angle transferring force to the output link, is an important force factor for all types of mechanisms. The ratio of velocities is reciprocal $\dot{S} / \dot{\sigma}=$ $\tan \theta$.

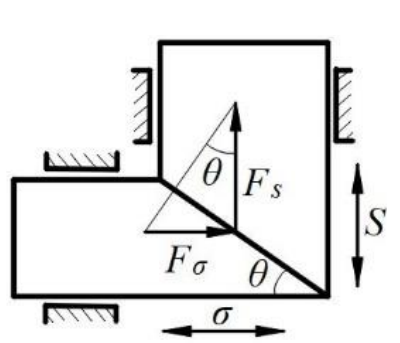

(a)

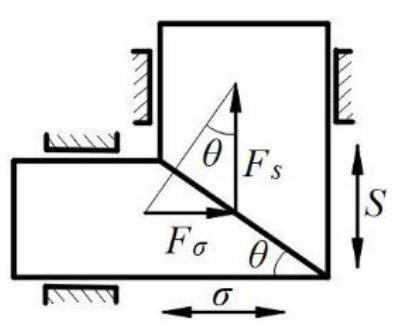

(c)

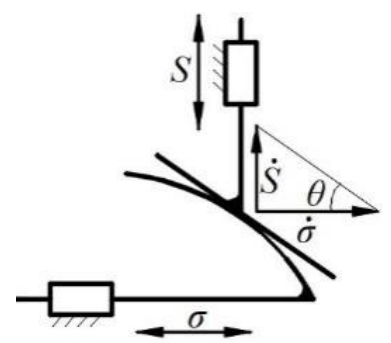

(b)

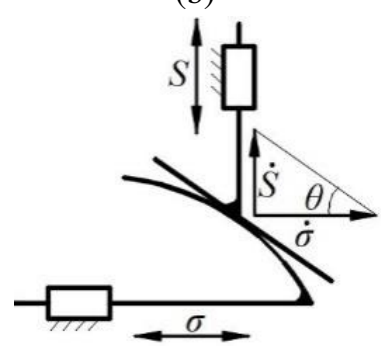

(d)

Fig. 1: Mechanism with two followers: (a) wedge; (b) CAM; (c) inverse CAM mechanism with a rectilinear groove of the output link; (d) inverse CAM mechanism with a curvilinear groove of the output link.

With the wedge mechanism (Fig. 1a) the edge contact between the movable members is almost inevitable, due to manufacturing inexactitude. Therefore, the contact surface of one of the units is rounded (Fig. 1b) or the unit ends with a roller (Fig. 1c), to reduce friction. In all three mechanisms, the output displacement $S=\sigma \tan \theta$ is proportional to the input transfer, because $\theta=$ const. The first transfer func- 
tion is $S^{\prime} \equiv d S / d \sigma=\tan \theta=$ const, therefore the second transfer function is $S^{\prime \prime} \equiv d^{2} S / d \sigma^{2}=0$.

Obviously, the mechanisms of Figs. 1a, 1b, and 1c cannot generate variable laws of motion. This is possible if the output link groove is curved, whereby a mechanism with profiled-follower is obtained. This is the so called inverse CAM mechanism (Fig. 1d).

\section{Synthesis of InVERse CAM Mechanism with TRANSLATing CAM AND FOLLOWER}

Mechanisms with translating CAM and translating follower are synthesized by assigned or deduced function $S=S(\sigma)$ by forced conditions and its first two derivatives $S^{\prime}=S^{\prime}(\sigma)$ and $S^{\prime \prime}=S^{\prime \prime}(\sigma)$, known as transfer functions. The input coordinate $\sigma \in\left(0, \sigma_{\max } \equiv H\right)$ is the shifting of the CAM, and the output coordinate $S \in\left(0, S_{\max } \equiv h\right)$ - the follower displacement. The angles $\beta$ and $\theta$ determine the output translation direction and the pressure angle from the roller to the output link, respectively (Fig. 2).

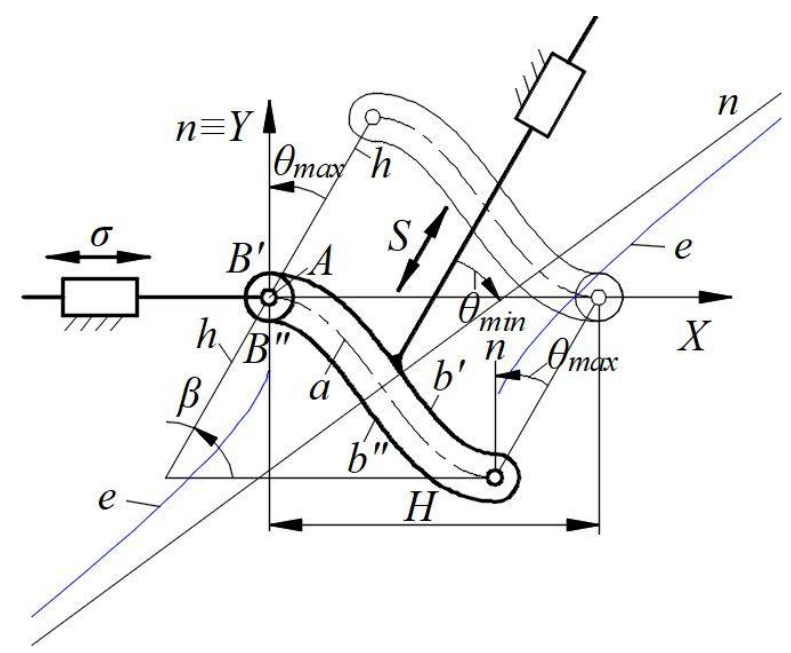

Fig. 2: Profiled-follower mechanism in start and in end position.

The coordinates, determining the position of the center $A$ of the roller that is mounted to the input drive slider shown in the $O X Y$ plane, defined by the profile member, are

$$
\begin{aligned}
& X_{A}=\sigma-S(\sigma) \cos \beta, \\
& Y_{A}=-S(\sigma) \sin \beta,
\end{aligned}
$$


describing curve of the center $a$ of the roller.

The angular coefficient of the normal $n$ to the pitch curve and to the equidistant center curve of the processing tools and the working CAM profiles, is determined by the derivative

$$
k_{n}=-\frac{d X_{A}}{d Y_{A}}=\frac{1-S^{\prime}(\sigma) \cos \beta}{S^{\prime}(\sigma) \sin \beta},
$$

where $S^{\prime}=d S / d \sigma$ is the first transfer function of the mechanism.

Pressure angle

$$
\theta=\arctan \frac{\tan \beta-k_{n}}{1+k_{n} \tan \beta}=\arctan \frac{S^{\prime}-\cos \beta}{\sin \beta}
$$

is the sharp angle locked between the direction of the output translation and the normal $n$.

Determining the angle $\beta$ from the condition $\theta_{\max }=-\theta_{\min }$ leads to $\min \theta_{\max }$, i.e. to the most advantageous transmission of force under predetermined conditions of synthesis. After equating the derivative of Eq. (4) to zero

$$
d \theta / d \sigma=S^{\prime \prime} \cos ^{2} \theta / \sin \beta
$$

is obtained the equation $S^{\prime \prime}(\sigma)=0$, from where the values of $\sigma$, for which both $\theta$ and $S^{\prime}$ have extremes. Absolute maximum values $S_{\max }^{\prime}$ and absolute minimum $S_{\min }^{\prime}$ of the $S^{\prime}$ are determined. If $S^{\prime \prime}(\sigma)$ does not become zero in the interval, the function $S^{\prime}(\sigma)$ is strictly monotonic. Then directly from $S^{\prime}(\sigma)$ are extracted $S_{\max }^{\prime}$ and $S_{\min }^{\prime}$.

By Eq. (4) a system of equations is formed

$$
\begin{aligned}
\tan \theta_{\text {min }} & =\frac{S_{\text {min }}^{\prime}-\cos \beta}{\sin \beta}, \\
\tan \theta_{\max } & =\frac{S_{\max }^{\prime}-\cos \beta}{\sin \beta},
\end{aligned}
$$

from which the angle is determined

$$
\beta=\arccos \frac{S_{\min }^{\prime}+S_{\max }^{\prime}}{2},
$$

that gives the direction of translation of the follower.

The travel of the translating roller $H \equiv \sigma_{\max }$ can be conditioned, for example, after selecting a driving force cylinder. In other cases, it can be defined according to constructive constraints, associated with the free space in which the mechanism is located. 
A minimum travel $H_{\min } \equiv \min \left(\sigma_{\max }\right)$ will be realized if determined by the condition $\theta_{\max }=-\theta_{\min }=\theta_{p}$, where $\theta_{p}$ is maximum permissible value $|\theta|$ of the pressure angle.

The second Eq. (6) is recorded in the form

$$
\tan \theta_{p}=\frac{k\left(S_{\max }^{\prime}-\cos \beta\right)}{\sqrt{1-k^{2} \cos ^{2} \beta}}
$$

where an unknown constant coefficient $k$ is introduced. With this multiplier the first transfer function $S_{m}^{\prime}=k S^{\prime}(\sigma)$ and the follower direction $\cos \beta_{m}=k \cos \beta$ is changed, because according to Eq. (7) the equivalence $k \cos \beta=0.5 k\left(S_{\max }^{\prime}+S_{\min }^{\prime}\right)$ is valid. From Eq. (8) is determined

$$
k=\frac{\tan \theta_{p}}{\sqrt{\left(\tan \theta_{p} \cos \beta\right)^{2}+\left(S_{\max }^{\prime}-\cos \beta\right)^{2}}},
$$

which makes it possible to calculate

$$
\beta_{m}=\arccos (k \cos \beta)
$$

and changed travel of the CAM

$$
H=\sigma_{\max } / k .
$$

The radius of the roller $r$, as it is known, must satisfy the conditions to

- avoid pointing of the output link curved profile;

- limit contact pressures;

- roll the roller along the profiled groove of the output link.

These conditions related to other types of CAM mechanisms, are given in details by Levitsky [1].

Also, it is known the condition for a more even wear of the roller, thereby finally determining the radius of the roller [9]. All points in the circular profile of the roller must be in contact with the work profile of the output unit an equal number of times. This means that the length of the circular profile of the roller has to be an integer, multiple of the active length of the curvilinear working profile of the output link.

The pitch curve of the roller in the plane of the output link is determined by Eq. (1). 
The center curves $b_{i}$ of the processing tools

$$
\begin{aligned}
& X_{B_{i}}=X_{B} \pm \operatorname{sign} k_{n} \frac{r_{i}-r}{\sqrt{1+k_{n}^{2}}}, \\
& Y_{B_{i}}=Y_{B} \pm k_{n}\left(X_{B_{i}}-X_{B}\right)
\end{aligned}
$$

are equidistant to the pitch curve of the roller, the normal distance $r_{i}-r$ being determined by the radius $r_{i}$ of a processing tool. When $r_{i}=0$ in Eq. (12), equations describing the curvilinear working profiles $b^{\prime}$ and $b^{\prime \prime}$ of the groove are obtained.

The evolute $e$ of the pitch curve of the roller of the equidistant curve of centers of the processing tools, and of the CAM profiles can be determined using parametric equations known from the differential geometry

$$
\begin{aligned}
& X_{A_{0}}(\sigma)=X_{A}(\sigma)-\frac{X^{\prime 2}{ }_{A}+Y_{A}^{\prime 2}}{X_{A}^{\prime}{ }_{A} Y_{A}^{\prime \prime}-Y_{A}^{\prime} X^{\prime \prime} A} Y_{A}^{\prime}(\sigma), \\
& Y_{A_{0}}(\sigma)=Y_{A}(\sigma)+\frac{X_{A}^{\prime 2}+Y_{A}^{\prime 2}}{X_{A}^{\prime}{ }_{A} Y^{\prime \prime}{ }_{A}-Y^{\prime}{ }_{A} X^{\prime \prime} A} X_{A}^{\prime}(\sigma),
\end{aligned}
$$

where

$$
\begin{aligned}
& X^{\prime}{ }_{A}=1-S^{\prime}(\sigma) \cos \beta, \quad X^{\prime \prime}{ }_{A}=-S^{\prime \prime}(\sigma) \cos \beta, \\
& Y^{\prime}{ }_{A}=-S^{\prime}(\sigma) \sin \beta, \quad Y^{\prime \prime}{ }_{A}=-S^{\prime \prime}(\sigma) \sin \beta
\end{aligned}
$$

are derived from the coordinates Eq. (1).

The radius of pitch curve of the roller may be determined by a known formula from the differential geometry:

$$
\rho_{A}(\sigma)=\frac{\left(X_{A}^{\prime 2}+Y_{A}^{\prime 2}\right)^{1.5}}{X_{A}^{\prime} Y^{\prime \prime}{ }_{A}-Y^{\prime}{ }_{A} X^{\prime \prime}{ }_{A}},
$$

or of the distance $l_{A_{0} A}$

$$
\rho_{A}(\sigma) \equiv l_{A_{0} A}=\sqrt{\left(X_{A}-X_{A_{0}}\right)^{2}+\left(Y_{A}-Y_{A_{0}}\right)^{2}} .
$$

The following is an example of synthesis of an inverse CAM mechanism with input translation and a profiled output link by a defined displacement function $S=$ $h u(\xi)$, profiled link travel $h \equiv S_{\max }=32$, and normalized function $u(\xi)=3 \xi^{2}-2 \xi^{3}$ with an argument $\xi=\in(0,1)$, defined by the ratio $\xi=\sigma / H$ of the input coordinate $\sigma \in\left(0, H \equiv \sigma_{\max }\right)$ to the travel $H$ of the input follower.

It is accepted $\theta_{p}=30^{\circ}$ and the maximum permissible CAM travel $H \equiv \sigma_{\max } \leq$ 80 in order CAM to be smaller. 
After differentiating the given function $S=h u(\xi)$ in regard to $\sigma$ the first and second transfer functions are derived: $S^{\prime}=6 h \xi(1-\xi) / H$ and $S^{\prime \prime}=6 h(1-2 \xi) / H^{2}$.

The inverse CAM mechanism will be synthesized under three different conditions:

1. An orthogonal position is set for the guides of the two movable links $\left(\beta=90^{\circ}\right)$. The travel of the input roller follower is looking for the requirement $|\theta|_{\max }=\theta_{p}$.

It was originally assumed that where the move can be chosen arbitrarily, $\sigma \in$ $\left(0, \sigma_{\max }\right)$, for example, the maximum permissible stroke $H=80$. Determined $S_{\max }^{\prime}=0.6$, it is substituted in Eq. (9) and the result $k=\tan \theta_{p} / S_{\max }^{\prime}=0.96225$ is replaced in Eq. (11), whereby the follower's travel is calculated: $H=\sigma_{\max } / k=$ 83.138 .

The value obtained for $H$ is unacceptable as it exceeds the maximum allowed value in the condition $H=80$.

2. The travel of the CAM is set to $H \equiv \sigma_{\max }=80$. An angle $\beta$ is sought for the most advantageous force transmission to the follower $\left(\theta_{\max }=-\theta_{\min }\right)$. Determined were $S_{\max }^{\prime}=0.6$ and $S_{\min }^{\prime}=0$, then they are substituted in Eqs. (6) and (7) from where $\theta_{\max }=-\theta_{\min }=17.457^{\circ}$ and $\beta=72.542^{\circ}$ are obtained, respectively.

3. Looking for the angle $\beta_{m}$ and the minimum travel $H_{\mathrm{min}}$ of the CAM from the condition of the most advantageous transmission of the force to the slide for $\theta_{\max }=-\theta_{\min }=\theta_{p}=30^{\circ}$. Initially, an arbitrarily value for the travel of the follower is assumed, for example maximum stroke $H=80$. The values $S_{\max }^{\prime}=0.6$ and $S_{\min }^{\prime}=0$ are substituted in Eq. (7) resulting in an angle $\beta=72.542^{\circ}$ which is substituted in Eq. (9). Calculated $k=1 / 0.6$, substituted in Eqs. (6) and (7), resulting in an angle $\beta_{m}=60^{\circ}$ and the travel of the input roller follower $H_{\min }=48$.

In accordance to the third condition, the mechanism presented in Fig. 2 is synthesized. The geometry of the mechanism is fully determined, including the common evolute of the CAM profiles and the equidistant center curves of the processing tools and the pitch curve of the roller.

\section{CONCLUSION}

The composite mathematical model for synthesis enables the geometry of inverse CAMs mechanism with translating CAM and translating follower to be fully determined. In addition to equations of the CAM profile and its equidistant center curves of the processing tools and the pitch curve roller, the following new results have been achieved:

1. The slope of the output follower is determined by a minimum absolute value of the pressure angle.

2. The minimal travel of the CAM is determined by a set permissible maximum value of the pressure angle. 
A specialized computer program for designing inverse CAMs mechanism with two followers by the so-developed mathematical model for synthesis was created.

There is a numerical example illustrating the application of the mathematical model under possible three different conditions of synthesis.

\section{REFERENCES}

[1] N.I. LeVitsky (1964) "CAM mechanisms". Mechanical engineering, Moscow (in Russian).

[2] F.Y. Chen (1982) "Mechanics and Design of CAM Mechanisms". Pergamon press, N. Y.

[3] H.A. Rothbart (1964) "CAMES. Design, Dynamics and Accuracy”, John Wiley \& Sons, N. Y.

[4] V. Galabov (1984) A Generalized Method for Kinematic Analysis and Synthesis of Plane Mechanisms. Journal of Theoretical and Applied Mechanics 14(2) 32-43.

[5] V. Galabov (1998) Structural and dimensional synthesis of mechanisms. Dissertation for D.Sc., TU-Sofia (in Bulgarian).

[6] P.W. Jensen (1987) "CAM Design and Manufacture". Marcel-Dekker Inc.

[7] V. Galabov (1980) Synthesis of CAM mechanisms with rectilinear motion of the CAM. Mechanical engineering 4 165-168 (in Bulgarian).

[8] R. Roussev, V. Galabov (2004) Synthesis of CAM mechanism with a translating CAM and a flat slotted lever. Yearbook of the Bulgarian Union of Scientists I 137-143 (in Bulgarian).

[9] R. Roussev, S. Savchev, N. Nikolov, V. Galabov (2005) Synthesis of CAM mechanism with a translating CAM and roller follower. Mechanics of machines 56 6468 (in Bulgarian).

[10] V. Galabov, V. DRaganov (1993) Synthesis of CAM-lever mechanisms for switches. Mechanics of machines 2 12-15 (in Bulgarian).

[11] J. Volmer (Hrsg) (1992) "Getriebelehre". Verlag Technik, Berlin.

[12] K.N. Hunt (1973) Profiled-Follower Mechanisms. Mechanism and Machine Theory 8 371-395.

[13] V. Galabov, D. Karagiozov (1987) Synthesis and application of mechanisms with profiled executive link. In: Proceedings of IFToMM Simpozium, Bulgaria 150-161.

[14] V. Galabov (1992) "Synthesis of mechanisms in robotics". Technical UniversitySofia, Sofia (in Bulgarian). 